\title{
Simultaneous Strain and Temperature Measurement Based on Chaotic Brillouin Optical Correlation-Domain Analysis in Large-Effective-Area Fibers
}

\author{
Xiaocheng ZHANG ${ }^{1,2}$, Shuangshuang $\mathrm{LIU}^{1,2}$, Jianzhong $\mathrm{ZHANG}^{1,2^{*}}$, \\ Lijun QIAO $^{1,2}$, Tao WANG ${ }^{1,2}$, Shaohua GAO $^{1,2}$, and Mingjiang ZHANG $^{1,2}$
}

\author{
${ }^{1}$ Key Laboratory of Advanced Transducers and Intelligent Control System of Ministry of Education, Taiyuan University \\ of Technology, Taiyuan 030024, China \\ ${ }^{2}$ College of Physics and Optoelectronics, Institute of Optoelectronic Engineering, Taiyuan University of Technology, \\ Taiyuan 030024, China \\ *Corresponding author: Jianzhong ZHANGＥ-mail: zhangjianzhong@tyut.edu.cn
}

\begin{abstract}
Chaotic Brillouin optical correlation domain analysis (BOCDA) has been proposed and experimentally demonstrated with the advantage of high spatial resolution. However, it faces the same issue of the temperature and strain cross-sensitivity. In this paper, the simultaneous measurement of temperature and strain can be preliminarily achieved by analyzing the two Brillouin frequencies of the chaotic laser in a large-effective-area fiber (LEAF). A temperature resolution of $1{ }^{\circ} \mathrm{C}$ and a strain resolution of $20 \mu \varepsilon$ can be obtained with a spatial resolution of $3.9 \mathrm{~cm}$. The actual temperature and strain measurement errors are $0.37^{\circ} \mathrm{C}$ and $10 \mu \varepsilon$, respectively, which are within the maximum measurement errors.
\end{abstract}

Keywords: Brillouin scattering; simultaneous strain and temperature measurement; chaotic laser; BOCDA; LEAF

Citation: Xiaocheng ZHANG, Shuangshuang LIU, Jianzhong ZHANG, Lijun QIAO, Tao WANG, Shaohua GAO, et al., "Simultaneous Strain and Temperature Measurement Based on Chaotic Brillouin Optical Correlation-Domain Analysis in Large-Effective-Area Fibers," Photonic Sensors, 2021, 11(4): 377-386.

\section{Introduction}

Brillouin optical correlation domain technology has been practically applied in strain monitoring of railways and bridges due to many advantages such as high spatial resolution and high sampling rate [1]. It can be mainly classified into two types: Brillouin optical correlation domain reflectometry (BOCDR) $[2,3]$ and Brillouin optical correlation domain analysis (BOCDA) [4-18]. Compared with the BOCDR technology, the BOCDA technology is based on stimulated Brillouin scattering (SBS), making it advantageous over BOCDR for long-range monitoring applications.

The BOCDA technology was proposed initially by Professor Hotate and his coauthor, where continuous-amplitude pump and probe waves were sinusoidal frequency modulated to realize distributed measurement of temperature and strain along the sensing fiber. Nevertheless, the BOCDA technology suffers from a trade-off between the measurement range and the spatial resolution [2]. To overcome the trade-off problem, some schemes such as time gating [5] and differential measurement [6]

Received: 12 July 2020 /Revised: 13 October 2020

(C) The Author(s) 2020. This article is published with open access at Springerlink.com

DOI: $10.1007 / \mathrm{s} 13320-020-0609-\mathrm{y}$

Article type: Regular 
have been employed at the cost of increased system complexity. Moreover, the continuous lightwave phase-modulated by a binary pseudorandom bit sequence (PRBS) or Golomb codes is also used as a detection signal to demodulate temperature and strain [7-12]. The range of unambiguous measurement in the basic configuration is restricted due to the periodicity of the correlation function. There are two ways to solve the problem of the ambiguous sensing distance. One is the combination between the correlation domain and the time domain for the simultaneous interrogation of a large number of correlation peaks [13]. The other is the utilization of the detection signal with only one correlation peak, such as amplified spontaneous emission [14], physical random code modulated laser [15], and chaotic laser [16-19]. The BOCDA technology reported so far can obtain the optimal measurement results with a sensing distance of $17.5 \mathrm{~km}$ and a spatial resolution of $8.3 \mathrm{~mm}$ [8]. However, the sensing mechanism of the BOCDA technology is based on the linear relationship between the Brillouin frequency shift and temperature or strain. Therefore, it faces the temperature and strain cross-sensitivity problem, which is a practical "bottleneck" of this sensing technology application.

To realize the simultaneous measurement of temperature and strain in the BOCDA technology, a dual-parameter method is proposed by using a $31-\mathrm{m}$ polarization maintaining fiber (PMF) [20, 21], where the birefringence-determined frequency deviation and Brillouin frequency shift have the different sign of strain and temperature dependence. The acquisition of the birefringence from the dynamic Brillouin grating makes the measurement system quite complex and the precise control of the polarization state also poses challenges for the measurement system to maintain a steady state [22]. In addition, a dual frequency shift method is utilized in a 4.74-m F-doped high-delta fiber, whose four different acoustic mode resonance frequencies are exploited to acquire the simultaneous measurement of temperature and strain [23]. Both of the above methods are aimed at the BOCDA technology with periodic correlation peaks, where the continuous wave with the sinusoidal frequency modulation is used as the detection signal. However, with regard to the BOCDA technology with one correlation peak, how to resolve the temperature and strain crosssensitivity issue has not yet been investigated till now.

In this paper, taking chaotic laser serving as the detection signal as an example, the simultaneous measurement of temperature and strain in the BOCDA technology with one correlation peak has been realized. By applying Peaks 1 and 3 of three Brillouin gain peaks of the chaotic laser in the large-effective-area fiber (LEAF), the measurement results with temperature error of $0.37^{\circ} \mathrm{C}$, the strain error of $10 \mu \varepsilon$, and a spatial resolution of $3.9 \mathrm{~cm}$ can be obtained.

\section{Principle of operation}

The chaotic pump and probe waves are injected into the optical fiber from both ends, respectively. The interference of two chaotic pumps and probe waves results in a traveling acoustic wave through the mechanism of electrostriction. The amplitude of the corresponding acoustic wave is proportional to the temporal cross correlation between the complex envelopes of the chaotic pump and probe waves given as [24]

$$
\begin{gathered}
\overline{Q(t, z)}=\frac{1}{2 \tau_{B}} \\
\int_{0}^{t} \exp \left(\frac{t^{\prime}-t}{2 \tau_{B}}\right) \overline{\left(t^{\prime}-\frac{z}{V_{g}}\right) A^{*}\left[t^{\prime}-\frac{z}{V_{g}}+\theta(z)\right]} \mathrm{d} t^{\prime}= \\
C[\theta(z)]
\end{gathered}
$$

where $A(t)$ is the complex envelope of the chaotic pump/probe wave, $V_{g}$ is the group velocity of light in the fiber, and $\tau_{B}$ is the acoustic wave lifetime. The time offset $\theta(z)$ is defined as $\theta(z)=(2 z-L) / V_{g}$, where $L$ is the fiber length. $C[\theta(z)]$ is the cross-correlation function between chaotic pump light and probe light. The triangle refractive index profile of LEAF makes it much more sensitive to 
temperature and strain [22]. Compared with ordinary single fiber (G.655), the mode field diameter of LEAF is $9.6 \mu \mathrm{m}$ in diameter larger than that of the G.655 fiber. As shown in the backscattering diagram of the LEAF in Fig. 1, it can be clearly seen that there are three Brillouin scattering peaks. The experimental conditions for obtaining the Brillouin scattering peaks are given in the following. The input fiber power is $10.01 \mathrm{~mW}$, and the length of the LEAF is $6 \mathrm{~km}$.

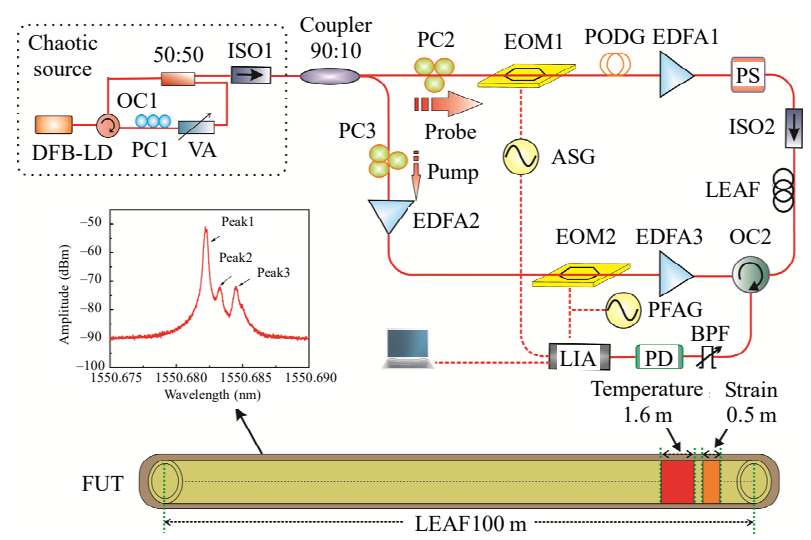

Fig. 1 Experimental setup of simultaneous strain and temperature measurement based on chaotic BOCDA. distributed-feedback laser diode (DFB-LD), variable attenuator (VA), polarization controllers ( $\mathrm{PC} 1, \mathrm{PC} 2$, and $\mathrm{PC} 3)$, electro-optic modulators (EOM1 and EOM2), programmable optical delay generator (PODG), erbium-doped optical fiber amplifiers (EDFA1, EDFA2, and EDFA3), analog signal generator (ASG), polarization scrambler (PS), isolators (ISO1 and ISO2), large effective area fiber (LEAF), optical circulators (OC1 and $\mathrm{OC} 2$ ), photodetector (PD), pulse function arbitrary generator (PFAG), optical band-pass filter (BPF), and lock-in amplifier (LIA).

Differences in composition or doping concentration in the LEAF core cause the longitudinal mode of the fiber to be different from the ordinary single-mode fiber, so that a multi-peak structure appears in the Brillouin spectrum. This may explain why each peak in LEAF has slightly different property, for Brillouin scattering depends on the strong correlation between the longitudinal acoustic and optical modes.

In the experiment, the Brillouin gain spectrum measured by the chaotic BOCDA system has three peaks. We take the first peak and the third peak for measurement. Theoretically, the Brillouin frequency shifts of the two peaks (Peaks 1 and 3) are

$$
\begin{gathered}
\Delta V_{B}^{\mathrm{PK} 1}=C_{\varepsilon}^{\mathrm{PK} 1} \Delta \varepsilon+C_{T}^{\mathrm{PK} 1} \Delta T \\
\Delta V_{B}^{\mathrm{PK} 3}=C_{\varepsilon}^{\mathrm{PK} 3} \Delta \varepsilon+C_{T}^{\mathrm{PK} 3} \Delta T .
\end{gathered}
$$

Just because the coefficients of temperature and strain are different for Peaks 1 and 3, the change of temperature and strain can be described as

$$
\begin{aligned}
\Delta T & =\frac{C_{\varepsilon}^{\mathrm{PK} 3} \Delta V_{B}^{\mathrm{PK} 1}-C_{\varepsilon}^{\mathrm{PK} 1} \Delta V_{B}^{\mathrm{PK} 3}}{C_{\varepsilon}^{\mathrm{PK} 3} C_{T}^{\mathrm{PK} 1}-C_{\varepsilon}^{\mathrm{PK} 1} C_{T}^{\mathrm{PK} 3}} \\
\Delta \mathcal{E} & =\frac{C_{T}^{\mathrm{PK} 1} \Delta V_{B}^{\mathrm{PK} 3}-C_{T}^{\mathrm{PK} 3} \Delta V_{B}^{\mathrm{PK} 1}}{C_{\varepsilon}^{\mathrm{PK} 3} C_{T}^{\mathrm{PK} 1}-C_{\varepsilon}^{\mathrm{PK} 1} C_{T}^{\mathrm{PK} 3}}
\end{aligned}
$$

where $\Delta T$ and $\Delta \varepsilon$ are the temperature and strain changes, respectively; $\Delta V_{B}^{\mathrm{PK} 1}$ and $\Delta V_{B}^{\mathrm{PK} 3}$ are the Brillouin frequency shifts of the first and third peaks of the Brillouin gain spectrum, respectively; $C_{T}{ }^{\mathrm{PK} 1}$ and $C_{T}{ }^{\mathrm{PK} 3}$ are the temperature coefficients of the first and third peaks, respectively; $C_{\varepsilon}{ }^{\mathrm{PK} 1}$ and $C_{\varepsilon}{ }^{\mathrm{PK} 3}$ are the strain coefficients of the first and third peaks, respectively.

\section{Experimental setup and results}

The experimental setup of simultaneously measuring temperature and strain based on the chaotic BOCDA is shown in Fig. 1. The chaotic laser source consists of a distributed-feedback laser diode without light isolators and a fiber feedback loop. The fiber feedback loop is composed of an optical circulator (OC1), a $3 \mathrm{~dB}$ optical coupler (50:50), a variable attenuator (VA), and a polarization controller ( $\mathrm{PC} 1)$. By adjusting the polarization state and feedback strength of the external feedback light, and the injection current, the system can generate the chaotic laser. The chaotic laser is divided into two beams by a $90 / 10$ coupler through an isolator (ISO1). One of them (90\%) through the light polarization controller (PC2) is injected into the electro-optic modulator (EOM1), which is used to suppress the carrier and double-sideband modulation, driven by the analog signal generator (ASG). The lower sideband output is utilized as the probe wave. Another beam $(10 \%)$ is amplified by an erbiumdoped fiber amplifier (EDFA2) after the light polarization controller (PC3), to be used as the pump wave. The probe signal modulated by the double 
sideband is transmitted through the variable optical delay lines (PODG, General Photonics ODG-101 and MDL-002). At this time, the optical signal is extremely weak, so the probe signal must be amplified by an EDFA1 to $11 \mathrm{dBm}$ and afterwards enters a polarization scrambler (PS). Here the PS can largely suppress polarization dependent gain fluctuations of the Brillouin signal. Then, the signal enters the fiber under test (FUT) through an optical isolator (ISO2). Since the pump path has only one-tenth of the optical input power and does not reach the operating power of the EOM2, an optical amplifier (EDFA2) is added in front of the EOM2 driven by the pulse function arbitrary generator (PFAG) with the sine wave. The pump signal is amplified to an average power of $32 \mathrm{dBm}$ by EDFA3 and injected into the FUT through the optical circulator (OC2). The SBS interaction between the pump and probe waves occurs in the FUT. Then, only the Stokes component of the probe wave via the SBS amplification is retained by a $6 \mathrm{GHz}$ optical band-pass filter (BPF). The probe signal is detected by a photodetector (PD) connected to a lock-in amplifier (LIA) for the signal processing. The lock-in amplifier reference frequency is provided by PFAG and its data acquisition is consistent with the ASG frequency sweeping. A 1.6-m-long temperature hot spot and a $0.5 \mathrm{~m}$ strain zone are placed at the end of the FUT, respectively.

The high spatial resolution of the chaotic BOCDA system is ensured by the low-coherence broadband chaotic light source. Figure 2 shows the autocorrelation trace of chaotic laser signals. The autocorrelation curve is obtained in the following procedure. Firstly, the chaotic signals from the chaotic light source are recorded by the real-time oscilloscope. Then according to (1), the autocorrelation curve is drawn by Matlab programming language in a personal computer. There is exclusively one main correlation peak in the chaotic autocorrelation trace, in which the SBS between the chaotic pump and probe waves occurs. The chaotic laser generated by the optical feedback system has an obvious time delay signature (TDS) due to the fixed external feedback cavity length. It can be seen that the chaotic autocorrelation trace has the secondary correlation peaks, which are the result of a weak amplitude autocorrelation of the chaotic signal occurring at the delay time of the external cavity. In our experiment, when the injection current is $18 \mathrm{~mA}$ and feedback strength is 0.115 (the feedback strength is defined as the ratio of the power of the feedback light to the output of the laser), we get the best chaotic state with the TDS suppression (i.e., the correlation coefficient is 0.098). Under such condition, the Brillouin gain spectra of the chaotic BOCDA system are effectively improved. The enlargement of the main correlation peak is further illustrated in the inset of Fig. 2. The spatial resolution is theoretically determined by full-width at half maximum of the main correlation peak. It can be seen that near the main peak, the autocorrelation trace has slight peak fluctuations. This is due to the laser relaxation oscillation. According to the Gaussian fitting peak width, the theoretical spatial resolution of the chaotic BOCDA system is $3.3 \mathrm{~cm}$.

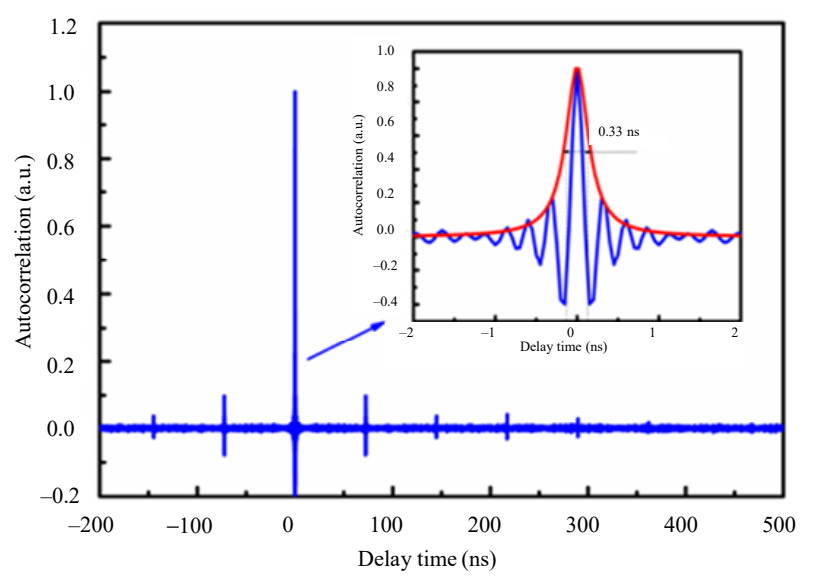

Fig. 2 Autocorrelation trace of the chaotic laser.

Figure 3(a) depicts the temperature dependence of the BGS with the strain-free in the FUT. The temperature is changed from $20^{\circ} \mathrm{C}$ to $40^{\circ} \mathrm{C}$ with the span of $5{ }^{\circ} \mathrm{C}$. It can be clearly observed that each BGS has three peaks with the central shifts of the first and third peaks moving from $10.5460 \mathrm{GHz}$ to $10.5680 \mathrm{GHz}$ and from 
$10.8330 \mathrm{GHz}$ to $10.8480 \mathrm{GHz}$, respectively. Figure 3(b) illustrates the temperature coefficient of the three peaks in the BGS. According to the temperature fitting curves, the first, second, and third peak temperature coefficients are $1.08 \mathrm{MHz} /{ }^{\circ} \mathrm{C}$, $1.10 \mathrm{MHz} /{ }^{\circ} \mathrm{C}$, and $0.73 \mathrm{MHz} /{ }^{\circ} \mathrm{C}$, respectively. Here, the first and third peaks are utilized to discriminate the temperature and strain in the experiment. This is because that the large difference in matric coefficients between the first and third peaks results in the high accuracy of temperature and strain measurement. We point out that it takes 3.26 minutes when using a lock-in amplifier to collect the BGS. This is because that for one gain spectrum, 601 points are needed to acquire and it is averaged 25 times. And the requiring time of each point is 0.013 seconds.

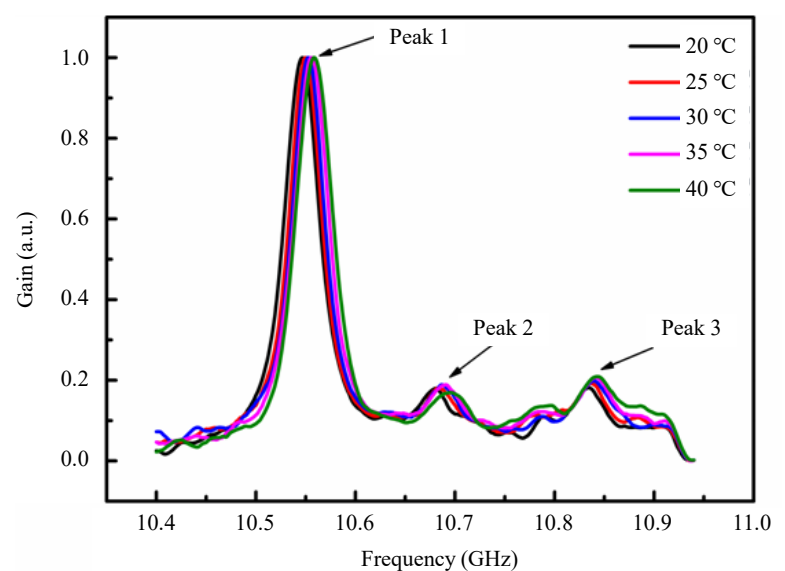

(a)

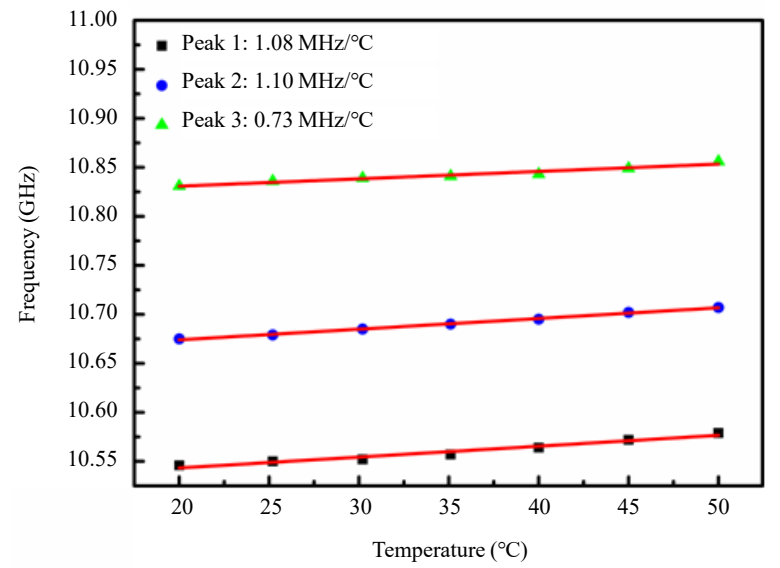

(b)

Fig. 3 Brillouin gain spectra of chaotic BOCDA in LEAF with different temperatures under (a) no strain and (b) the temperature-dependence coefficient of Brillouin frequency shift for the three peaks.
Figure 4(a) shows the strain dependence of the BGS with the temperature-free in the FUT. We can clearly observe that when the LEAF is kept at a constant temperature $\left(20^{\circ} \mathrm{C}\right)$, the strain is changed from 0 to $1000 \mu \varepsilon$ with the measurement interval of $200 \mu \varepsilon$. The center frequency shift of the first peak moves from $10.5460 \mathrm{GHz}$ to $10.6010 \mathrm{GHz}$, and the center frequency shift of the third peak moves from $10.8330 \mathrm{GHz}$ to $10.8830 \mathrm{GHz}$.

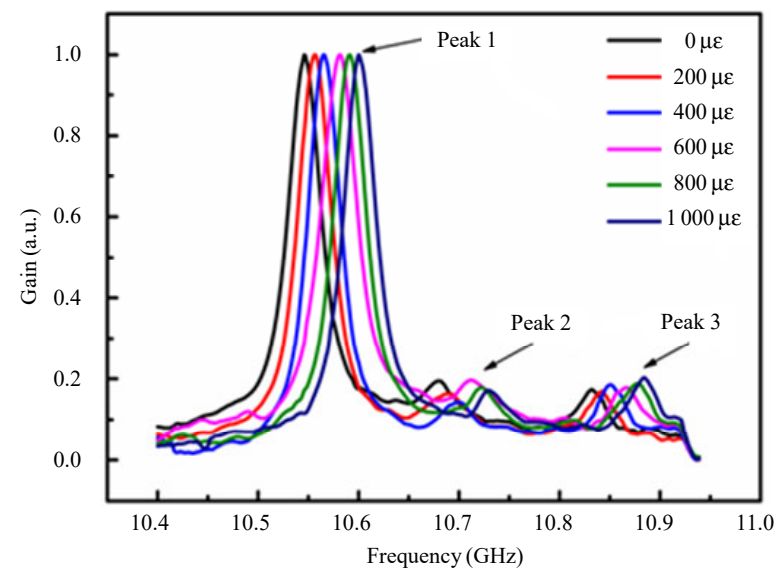

(a)

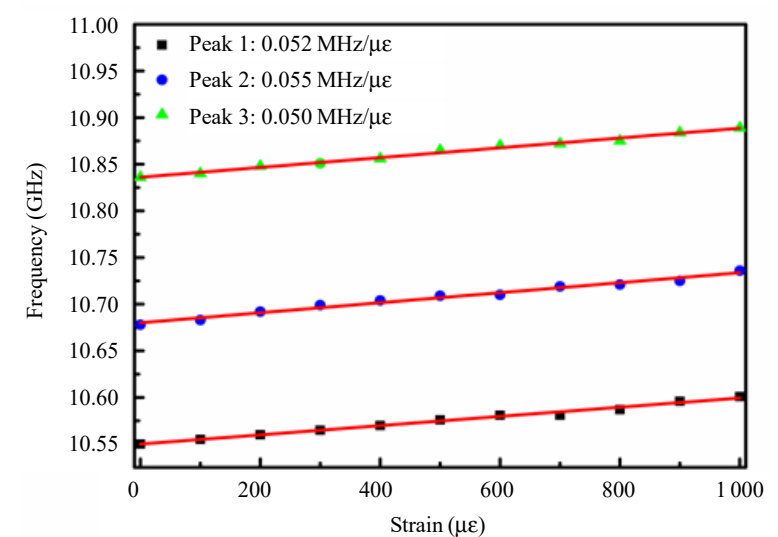

(b)

Fig. 4 Brillouin gain spectra of chaotic BOCDA in LEAF with different strains under (a) no temperature and (b) the strain-dependence coefficient of Brillouin frequency shift for the three peaks.

The strain coefficients of the three peaks are shown in Fig. 4(b). The strain fitting curve shows that the first, second and third peak strain coefficients are $0.052 \mathrm{MHz} / \mu \varepsilon, 0.055 \mathrm{MHz} / \mu \varepsilon$, and $0.050 \mathrm{MHz} / \mu \varepsilon$, respectively. The uncertainty of temperature and strain is about $0.5 \mathrm{MHz}$ by calculating the maximum standard deviation of 
Brillouin frequency shift, which is repeated 25 times [17].

Figure 5 shows the measured BFS distributions along the FUT. It can be seen that each peak has obvious BFS distribution. The structure of the FUT is made up of a $100 \mathrm{~m}$ LEAF, in which a $1.6 \mathrm{~m}$ near $92 \mathrm{~m}$ is placed in a fiber thermostat $\left(55^{\circ} \mathrm{C}\right)$ with a loose state and a $0.5 \mathrm{~m}$ near $96 \mathrm{~m}$ is stretched to be $1000 \mu \varepsilon$ with no temperature change. Other section of the LEAF is maintained at room temperature $\left(27^{\circ} \mathrm{C}\right)$ with a loose state. We can also see that at the heated position, the BFSs of the first and third peaks are changed from $10.5530 \mathrm{GHz}$ to $10.5830 \mathrm{GHz}$ and from $10.8380 \mathrm{GHz}$ to $10.8585 \mathrm{GHz}$, respectively. At the stretching location, the BFSs of the first and third peaks have the changes from $10.5530 \mathrm{GHz}$ to $10.6050 \mathrm{GHz}$ and from $10.8380 \mathrm{GHz}$ to $10.8880 \mathrm{GHz}$, respectively.

To more intuitively display the BGS distribution, the three-dimensional view of the measured BGS along the FUT is further shown in Fig. 6. The LEAF is heated and stretched in the same way as Fig. 5. The BFSs of the hot spot area have significant frequency shifts of approximately $31 \mathrm{MHz}$ and $21 \mathrm{MHz}$ for the first and third peaks respectively, when the temperature of the hot area is increased to be $55^{\circ} \mathrm{C}$ from the room temperature. For the strain area, the first and third peak BFSs are about $52 \mathrm{MHz}$ and $50 \mathrm{MHz}$, respectively, when the strain of the stretched section is set to $1000 \mu \varepsilon$.

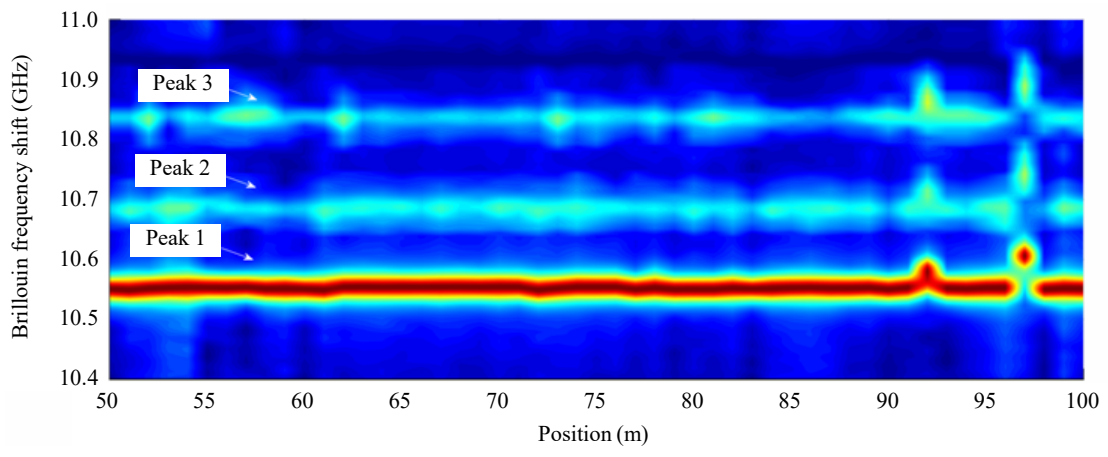

Fig. 5 BFS distributions along the LEAF in the chaotic BOCDA system. Along a $100 \mathrm{~m}$ LEAF, a $1.6 \mathrm{~m}$ near $92 \mathrm{~m}$ is placed in a fiber thermostat $\left(55^{\circ} \mathrm{C}\right)$ with a loose state and a $0.5 \mathrm{~m}$ near $96 \mathrm{~m}$ is stretched to be $1000 \mu \varepsilon$ with no temperature change.

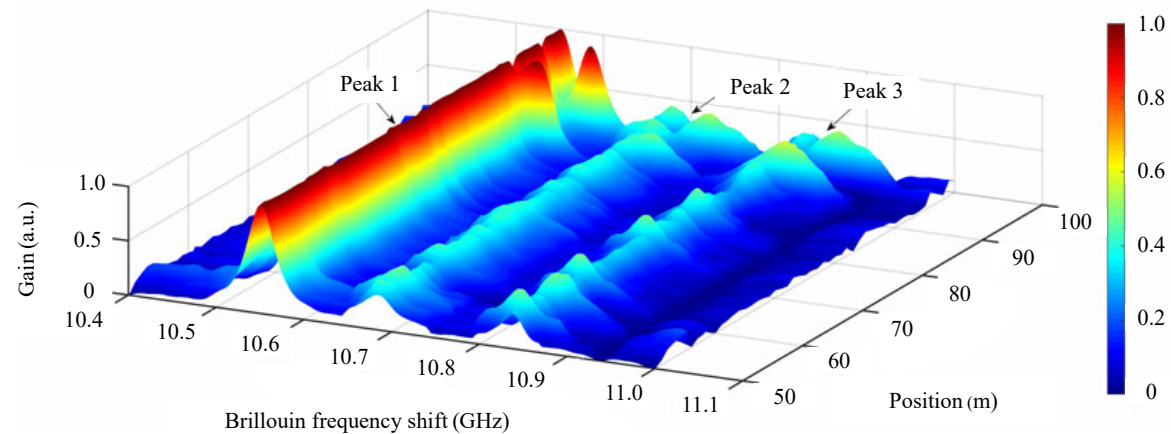

Fig. 6 Three-dimensional plot of the measured BGS distribution along the LEAF in chaotic BOCDA system. The LEAF is heated and stretched in the same way as Fig. 5.

The measured BFSs distributions of the first and third peaks along the FUT using the chaotic BOCDA technology are shown in Fig. 7. The spatial resolution of the chaotic BOCDA system can be measured by the average value of $10 \%-90 \%$ of the rise and fall time equivalent lengths in meter for the temperature and stretched section. We can clearly observe that the $1.6 \mathrm{~m}$ section is the temperature region and the $0.5 \mathrm{~m}$ section is the strain region. The rise and fall time equivalent lengths for the temperature region are $3.8 \mathrm{~cm}$ and $4.0 \mathrm{~cm}$, respectively. The rise and fall time equivalent 
lengths for the strain region are $3.5 \mathrm{~cm}$ and $3.9 \mathrm{~cm}$, respectively. Therefore, the spatial resolution can approximately approach $3.9 \mathrm{~cm}$ along the $100 \mathrm{~m}$ LEAF, which is almost in line with the theoretical spatial resolution of $3.3 \mathrm{~cm}$ obtained from Fig. 2 .

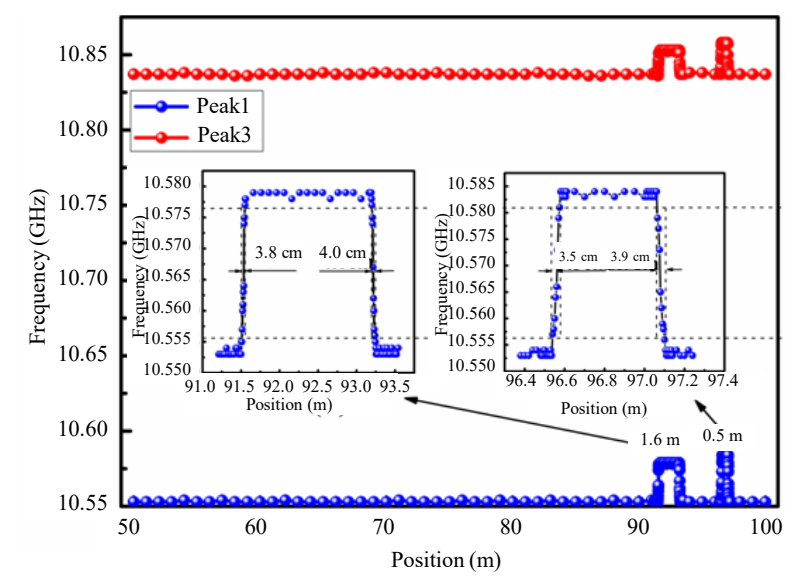

Fig. 7 Measured BFS distributions of the first and third peaks along the FUT.

We note that the BGS shown in Fig. 5, the effect of the heated section looks very gradual and does not appear to change abruptly in position. However, from Fig. 7, the $3.9 \mathrm{~cm}$ spatial resolution can be achieved. Here, we explain why an abruptly changing peak from a gradually changing spectrum can be extracted. This is because the obtained sampling points in Figs. 5 and 7 are different. The function of Fig. 5 is to demonstrate that our scheme can achieve the distributed measurement along the LEAF. Therefore, 100 points along LEAF are taken, i.e., one point per meter. Thus for the temperature zone of $1.6 \mathrm{~m}$, there is only one BGS in the temperature zone. So, the effect of the heated section looks very gradual. The function of Fig. 7 is to prove the spatial resolution of our experimental system. Near the hot spot, one point per $5 \mathrm{~mm}$ is taken and 60 points are accessed. If Fig. 5 has the same sampling points near the hot zone with Fig.7, they have the same changing trend.

Finally, we investigate the decoupling capability of the temperature and strain of the chaotic BOCDA system. When the strain of $1000 \mu \varepsilon$ and the heat of $55^{\circ} \mathrm{C}$ are imposed on two sections of the LEAF simultaneously, the corresponding BFSs are obtained, as show in Fig. 5. Here, the first and third peaks are utilized to discriminate the temperature and strain, taking the high accuracy of temperature and strain measurement into account. According to (4) and (5), the temperature and strain simultaneous measurement can be successfully achieved. The decoupling result of the temperature and strain along the LEAF is illustrated in Fig. 8. The red and blue dot lines represent the demodulated temperature and strain distribution, respectively. The demodulated temperature and strain agree well with the actual values, respectively. Of course, we can also see that some error fluctuations exit in the experimental measurement, but these are within the acceptable maximum error.

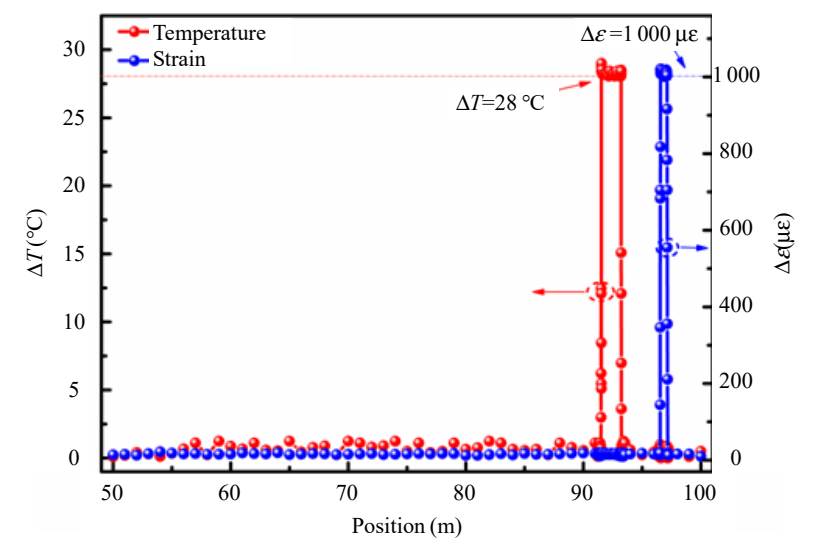

Fig. 8 Temperature (red dots) and strain (blue dots) decoupling result along the LEAF.

\section{Discussion}

In this section, the error analysis for the simultaneous measurement of strain and temperature is performed in detail. Firstly, according to the error analysis reported by Jones [25], the temperature error $\delta T$ and strain error $\delta \varepsilon$ of the chaotic BOCDA system are given as follows:

$$
\begin{gathered}
\delta T=\frac{\left|C_{\varepsilon}^{\mathrm{PK} 3}\right| \delta v^{\mathrm{PK} 1}+\left|C_{\varepsilon}^{\mathrm{PK} 1}\right| \delta v^{\mathrm{PK} 3}}{\left|C_{T}^{\mathrm{PK} 3} C_{\varepsilon}^{\mathrm{PK} 1}-C_{\varepsilon}^{\mathrm{PK} 3} C_{T}^{\mathrm{PK} 1}\right|} \\
\delta \varepsilon=\frac{\left|C_{T}^{\mathrm{PK} 3}\right| \delta v^{\mathrm{PK} 1}+\left|C_{\varepsilon}^{\mathrm{PK} 1}\right| \delta v^{\mathrm{PK} 3}}{\left|C_{T}^{\mathrm{PK} 3} C_{\varepsilon}^{\mathrm{PK} 1}-C_{\varepsilon}^{\mathrm{PK} 3} C_{T}^{\mathrm{PK} 1}\right|}
\end{gathered}
$$


where $\delta v^{\mathrm{PK} 1}$ and $\delta v^{\mathrm{PK} 3}$ are the BFS errors of the first and third peaks, respectively. Putting above strain/temperature coefficients into (6) and (7), and taking the BFS errors of our measurement system $\left(\delta v^{\mathrm{PK} 1}=0.5 \mathrm{MHz}\right.$ and $\left.\delta v^{\mathrm{PK} 3}=0.5 \mathrm{MHz}\right)$ into account, we find that the maximum temperature and strain errors are $3.18^{\circ} \mathrm{C}$ and $56 \mu \varepsilon$, respectively.

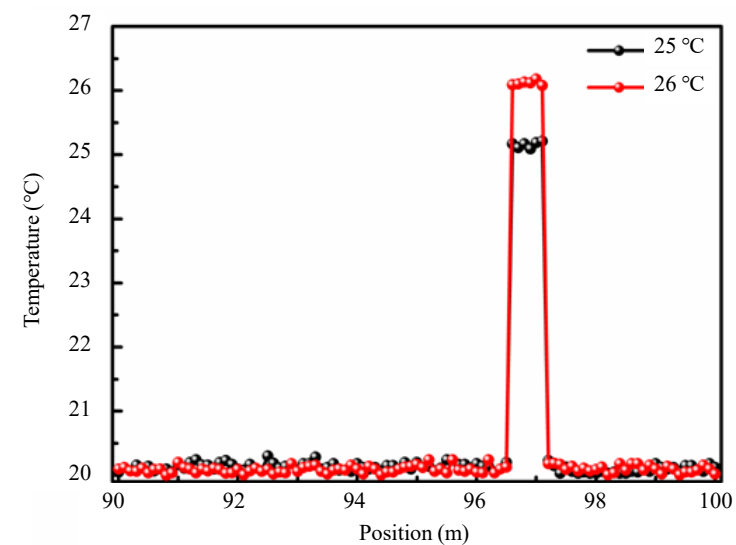

(a)

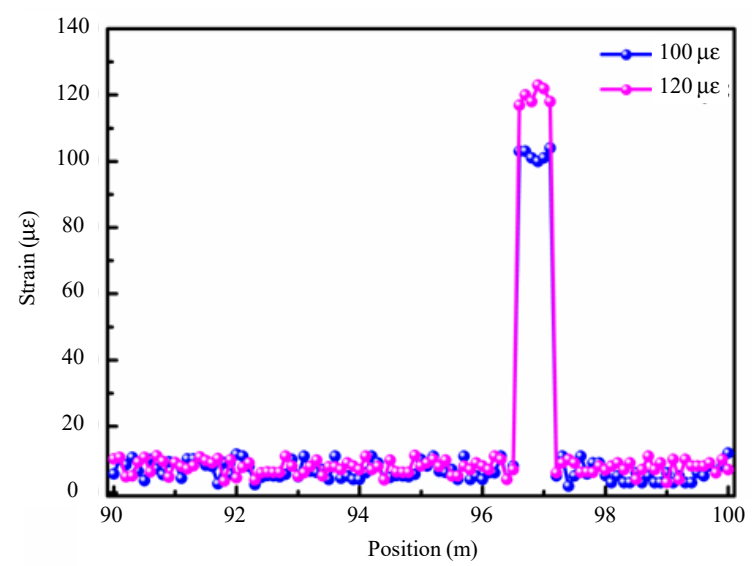

(b)

Fig. 9 Temperature and strain $\left(25{ }^{\circ} \mathrm{C} / 100 \mu \varepsilon\right.$ and $\left.26^{\circ} \mathrm{C} / 120 \mu \varepsilon\right)$ are applied on the section of the test LEAF, and (a) the temperature difference $\left(1^{\circ} \mathrm{C}\right)$ and (b) the strain difference $(20 \mu \varepsilon)$ can be distinguishable.

When two sets of temperature and strain $\left(25^{\circ} \mathrm{C} / 100 \mu \varepsilon\right.$ and $\left.26^{\circ} \mathrm{C} / 120 \mu \varepsilon\right)$ are applied on the section of the test LEAF, the simultaneous temperature and strain measurement comparisons are made. This comparison also demonstrates the complete discrimination ability of our proposed method. And the temperature difference $\left(1^{\circ} \mathrm{C}\right)$ and the strain difference $(20 \mu \varepsilon)$ can be distinguishable. From Fig. 9, the temperature and strain for reference are $20^{\circ} \mathrm{C}$ and $0 \mu \varepsilon$, respectively. When $25^{\circ} \mathrm{C}$ and $100 \mu \varepsilon$ are set, the measured BFSs for Peaks 1 and 3 are $10.5560 \mathrm{GHz}$ and $10.8415 \mathrm{GHz}$, respectively. When $26^{\circ} \mathrm{C}$ and $120 \mu \varepsilon$ are set, the measured BFSs of Peaks 1 and 3 are $10.5580 \mathrm{GHz}$ and $10.8430 \mathrm{GHz}$, respectively. The corresponding demodulated temperature and strain are shown in Figs. 9(a) and 9(b), respectively. According to (4) and (5), the calculated temperature and strain changes are $1.37^{\circ} \mathrm{C}$ and $10 \mu \varepsilon$, respectively. Therefore, we get the actual measurement temperature and strain errors are $0.37^{\circ} \mathrm{C}$ and $10 \mu \varepsilon$, which are within the maximum measurement errors.

\section{Conclusions}

In general, we have successfully used the chaotic BOCDA system to simultaneously measure temperature and strain. As the frequency shifts of the first and third peaks of the BGS in the LEAF have a different linear relationship with strain and temperature, the temperature difference $\left(1^{\circ} \mathrm{C}\right)$ and the strain difference $(20 \mu \varepsilon)$ can be distinguishable. Preliminary experimental results indicate that a spatial resolution of $3.9 \mathrm{~cm}$ within the $100-\mathrm{m}$ sensing distance can be achieved. The temperature and strain are measured simultaneously with a strain error of $10 \mu \varepsilon$ and a temperature error of $0.37^{\circ} \mathrm{C}$. With the successful resolution of the temperature and strain cross-sensitivity problem, the sensing system based on chaotic BOCDA in LEAFs will have a great potential in practical structural health monitoring, such as large civil bridges and dams.

\section{Acknowledgment}

This work was supported in part by the National Natural Science Foundation of China (NSFC) (Grant Nos. 61527819 and 61875146), in part by the Research Project Supported by Shanxi Province Youth Science and Technology Foundation (Grant No. 201601D021069), in part by the Key Research and Development Program (High-Tech Field) of Shanxi Province (Grant Nos. 201803D121064 and 
201803D31044), in part by the Program for Sanjin Scholar, in part by the Transformation of Scientific and Technological Achievements Programs (TSTAP) of Higher Education Institutions in Shanxi, and in part by the Program for the Outstanding Innovative Teams of Higher Learning Institutions of Shanxi.

Open Access This article is distributed under the terms of the Creative Commons Attribution 4.0 International License (http://creativecommons.org/licenses/by/4.0/), which permits unrestricted use, distribution, and reproduction in any medium, provided you give appropriate credit to the original author(s) and the source, provide a link to the Creative Commons license, and indicate if changes were made.

\section{References}

[1] H. J. Yoon, K. Y. Song, C. Y. Choi, H. S. Na, and J. S. Kim, "Real-time distributed strain monitoring of a railway bridge during train passage by using a distributed optical fiber sensor based on Brillouin optical correlation domain analysis," Journal of Sensors, 2016, 2016: 9137531.

[2] Y. Mizuno, W. W. Zou, Z. Y. He, and K. Hotate, "Proposal of Brillouin optical correlation-domain reflectometry (BOCDR)," Optics Express, 2008, 16(16): 12148-12153.

[3] Y. Mizuno, H. Lee, and K. Nakamura, "Recent advances in Brillouin optical correlation-domain reflectometry," Applied Sciences, 2018, 8(10): 1845.

[4] K. Hotate and T. Hasegawa, "Measurement of Brillouin gain spectrum distribution along an optical fiber using a correlation-based technique-proposal, experiment and simulation," IEICE Transactions on Electron, 2000, 83(3): 405-412.

[5] Y. Mizuno, Z. Y. He, and K. Hotate, "Measurement range enlargement in Brillouin optical correlation domain reflectometry based on temporal gating scheme," Optics Express, 2009, 17(11): 9040-9046.

[6] J. H. Jeong, K. Lee, K. Y. Song, J. M. Jeong, and S. B. Lee, "Differential measurement scheme for Brillouin optical correlation domain analysis," Optics Express, 2012, 20(24): 27094-27101.

[7] A. Zadok1, Y. Antman, N. Primerov, A. Denisov, J. Sancho, and L. Thevenaz, "Random-access distributed fiber sensing," Laser \& Photonics Reviews, 2012, 6(5): 1-5.

[8] A. Denisov, M. A. Soto, and L. Thevenaz, "Going beyond 1000000 resolved points in a Brillouin distributed fiber sensor: theoretical analysis and experimental demonstration," Light: Science \& Applications, 2016, 5: e16074.

[9] Y. London, Y. Antman, E. Preter, N. Levanon, and A.
Zadok, "Brillouin optical correlation domain analysis addressing 440000 resolution points," Journal of Lightwave Technology, 2016, 34(19): 4421-4429.

[10] O. Shlomi, E. Preter, D. Ba, Y. London, Y. Antman, and A. Zadok, "Double-pulse pair Brillouin optical correlation-domain analysis," Optics Express, 2016, 24(23): 26867-26876.

[11] M. Matsumoto and S. Akai, "High-spatial-resolution Brillouin optical correlation domain analysis using short-pulse optical sources," Journal of Lightwave Technology, 2019, 37(24): 6007-6014.

[12] D. X. Ba, Y. Li, J. L. Yan, X. P. Zhang, and Y. K. Dong, "Phase-coded Brillouin optical correlation domain analysis with $2-\mathrm{mm}$ resolution based on phase-shift keying," Optics Express, 2019, 27(25): 36197.

[13] D. Elooz, Y. Antman, N. Levanon, and A. Zadok, "High resolution long reach distributed Brillouin sensing based on combined time-domain and correlation-domain analysis," Optics Express, 2014, 22(6): 6454-6463.

[14] R. Cohen, Y. London, Y. Antman, and A. Zadok, "Brillouin optical correlation domain analysis with 4 millimeter resolution based on amplified spontaneous emission," Optics Express, 2014, 22(10): 12070-12078.

[15] M. W. Li, X. C. Zhang, J. Z. Zhang, J. G. Zhang, M. J. Zhang, L. J. Qiao, et al., "True random coding for Brillouin optical correlation domain analysis," OSA Continuum, 2019, 2(7): 2234-2243.

[16] J. Z. Zhang, M. T. Zhang, M. J. Zhang, Y. Liu, C. K. Feng, Y. H. Wang, et al., "Chaotic Brillouin optical correlation-domain analysis," Optics Letters, 2018, 43(8): 1722-1725.

[17] J. Z. Zhang, C. K. Feng, M. J. Zhang, Y. Liu, C. Y. $\mathrm{Wu}$, and Y. H. Wang, "Brillouin optical correlation domain analysis based on chaotic laser with suppressed time delay signature," Optics Express, 2018, 26(6): 6962-6972.

[18] J. Z. Zhang, Y. H. Wang, M. J. Zhang, Q. Zhang, M. W. Li, C. Y. Wu, et al., "Time-gated chaotic Brillouin optical correlation domain analysis," Optics Express, 2018, 26(13): 17597-17607.

[19] Y. H. Wang, L. Zhao, M. J. Zhang, J. Z. Zhang, L. J. Qiao, T. Wang, et al., "Dynamic strain measurement by a single-slope-assisted chaotic Brillouin optical correlation-domain analysis," Optics Letters, 2020, 45(7): 1822-1825.

[20] W. Zou, Z. Y. He, and K. Hotate, "Complete discrimination of strain and temperature using Brillouin frequency shift and birefringence in a polarization-maintaining fiber," Optics Express, 2009, 17(3): 1248-1255.

[21] W. Zou, Z. Y. He, and K. Hotate, "Demonstration of Brillouin distributed discrimination of strain and temperature using a polarization-maintaining optical 
fiber," IEEE Photonics Technology Letters, 2010, 22(8): 526-528.

[22] X. Liu and X. Y. Bao, "Brillouin spectrum in LEAF and simultaneous temperature and strain measurement," Journal of Lightwave Technology, 2012, 30(8): 1053-1059.

[23] W. Zou, Z. Y. He, M. Kishi, and K. Hotate, "Stimulated Brillouin scattering and its dependences on strain and temperature in a high-delta optical fiber with F-doped depressed inner cladding," Optics
Letters, 2007, 32(6): 600-602.

[24] R. Cohen, Y. London, Y. Antman, and A. Zadok, "Brillouin optical correlation domain analysis with 4 millimeter resolution based on amplified spontaneous emission," Optics Express, 2014, 22(10): 12070-12078.

[25] J. D. C. Jones, "Review of fibre sensor techniques for temperature-strain discrimination," in 12th International Conference on Optical Fiber Sensors, America, October 28, 1997, pp: 36-39. 